\title{
Organic Photovoltaic Materials
}

- Design, Synthesis and Scale-

\section{up}

Wallace W. H. Wong, ${ }^{*[a]}$ James L. Banal, ${ }^{[a]}$ Paul B. Geraghty, ${ }^{[a]}$ Quentin Hong, ${ }^{[a]}$ Bolong Zhang, ${ }^{\text {[a] }}$ Andrew B. Holmes ${ }^{[a]}$ and David J. Jones ${ }^{[a]}$

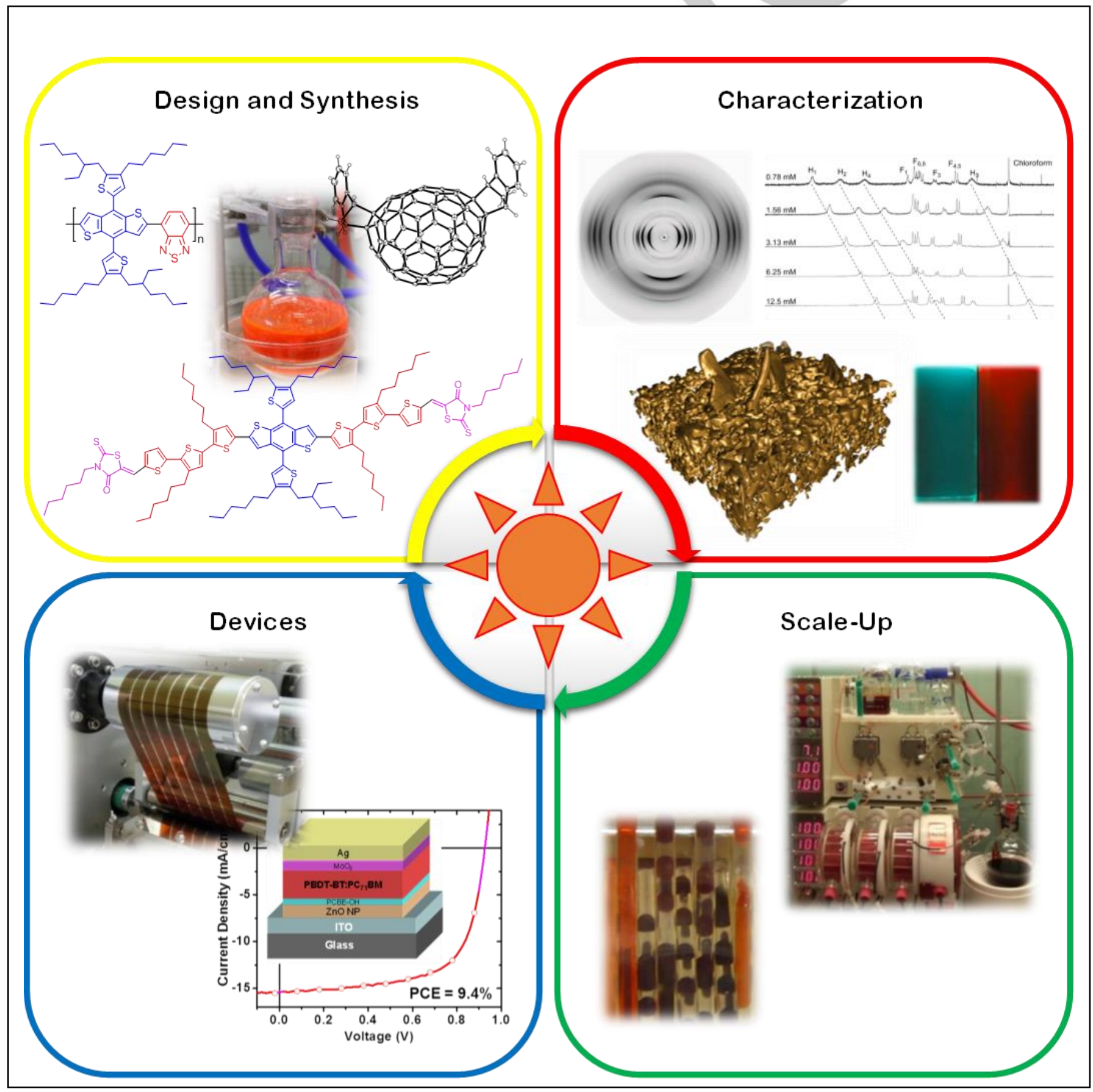


Abstract: This account describes the work of our group in the area of organic photovoltaics in the past six years. The emphasis is on our experiences in the development of the organic materials, their characterization, scale-up and application in devices. We share our insight into the relationship between synthetic methods, molecular properties, bulk material properties and device performance.

\section{Organic Photovoltaics}

The field of organic photovoltaics (OPV) has been an area of intense investigation for researchers with expertise in materials chemistry, photophysics and engineering. Progress was slow in the early studies of the 1980s and 1990s as much less was known about the details of device operation mechanism and materials design requirements. As the level of knowledge and expertise accumulated across the disciplines, significant improvements have been achieved in device efficiency as well as stability. Advancing the state-of-the-art is a great challenge as it is only possible through a combination of high performance material and careful device optimization and processing.

\subsection{State-of-the-art}

Power conversion efficiency (PCE) is the most commonly assessed metric for OPV materials and devices. The significance of work in the area is often judged by the PCE values presented whether or not it is done fairly or with intention. This drives research groups to trial and error experimentation approach to target high PCE numbers as quickly as possible. In this account, the focus is on bulk heterojunction (BHJ) materials and devices where the benchmark PCE is just over $10 \% .{ }^{[1-3]}$ While development in device architecture and assembly is an essential ingredient to achieve state-of-the-art, we want to share here our experiences on materials design, synthesis, characterization and scale-up. Readers can refer to reviews on polymers ${ }^{[4-10]}$ and molecular materials ${ }^{[11-13]}$ for more comprehensive surveys of the literature.

\subsection{Materials design requirements}

Designing OPV materials from scratch is a complex problem. ${ }^{[14]}$ The essential components of the active layer of a BHJ device are compatible electron donor and electron acceptor materials. Achieving material compatibility is relatively straightforward as orbital energies can be controlled by structural modification guided by theoretical calculations dictated from the frontier orbital state energy perspective (Figure 1). Similarly, desirable photo-

[a] Dr W. W. H. Wong, Mr J. L. Banal, Mr P. B. Geraghty, Mr Q. Hong, Mr B. Zhang, Prof A. B. Holmes, Dr D. J. Jones.

School of Chemistry

University of Melbourne

Bio21 Institute, 30 Flemington Road, Parkville, Victoria, 3010,

Australia.

E-mail: wwhwong@unimelb.edu.au physical properties can also be achieved through molecular structure design. On the other hand, bulk material properties, such as solvent solubility, thermal properties, crystallinity and semiconducting behavior, can be hard to target with any degree of accuracy. Yet, bulk properties are every bit as important as molecular properties in determining device performance. This is especially the case for $\mathrm{BHJ}$ devices where the mixing and phase separation of donor and acceptor materials must result in the formation of nanoscale interpenetrating networks for efficient device operation (Figure 1). Certainly, correlation between molecular and bulk properties and device performance has been observed in many studies but it takes a brave scientist to predict the performance of new sets of materials. In this account, we present some of our studies on polymer design and synthesis, continuous flow processing and various molecular materials. These studies gave a variety of both predictable and unexpected outcomes.

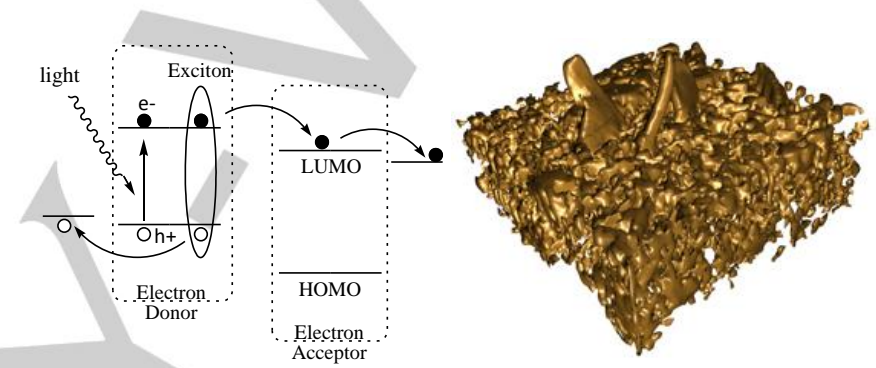

Figure 1. Simplified state energy level diagram showing the highest occupied molecular orbital (HOMO) and lowest unoccupied molecular orbital (LUMO) energy level offset required for the electron donor and acceptor materials and the process of excitation and charge separation in bulk heterojunction devices ${ }^{[0]}$ and an illustration of a nanoscale phase separated active layer generated from transmission electron microscopy (TEM) tomography data. ${ }^{[15]}$

Dr Wallace W. H. Wong is an Australian Research Council Future Fellow at the University of Melbourne. He has been working in the area of organic electronic materials since 2006. His area of research interests include semiconducting polymers, fullerene chemistry, luminescent dyes, continuous flow processing and supramolecular chemistry.

James Banal is a PhD candidate at the University of Melbourne working on luminescent solar concentrator materials. $\mathrm{He}$ completed his bachelor's degree in 2011 at the University of Santo Tomas, Philippines with a thesis on conducting polymer chemical sensors. In 2012, he graduated with first-class honours at the University of Melbourne working on electron deficient conjugated polymers.

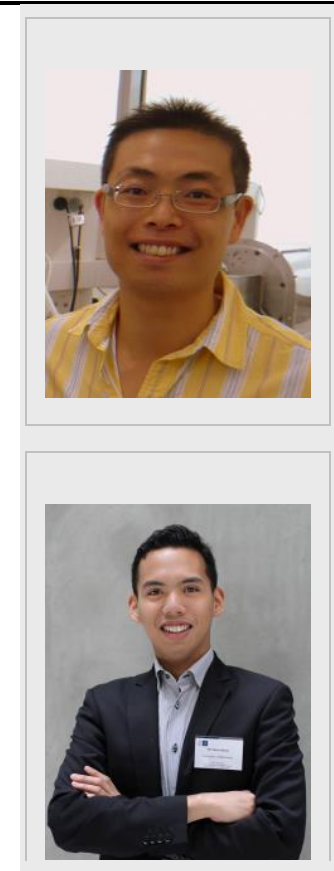


Paul B. Geraghty is a PhD candidate at the University of Melbourne. He is investigating the synthesis and degradation properties of high performance organic electronic materials. He completed a B.Sc. in 2010 and graduated with an M.Sc (Hons) in 2013 both from the University of Canterbury, New Zealand.

Quentin Hong is a PhD student at the University of Melbourne. He received his Bachelor's degree in Chemistry from the same university and was awarded the Australian Postgraduate Award to study under the tutelage of Dr. Wallace Wong. Currently his research interests is focussed on developing supramolecular assemblies for the use in organic electronics.

Bolong Zhang is a Masters degree student at the University of Melbourne. He has been working in the area of organic electronic materials since 2013. His area of research interests include fullerene functionalization and photovoltaic device fabrication.

Prof Andrew B. Holmes is Laureate Professor Emeritus in the School of Chemistry, Bio21 Institute and Emeritus Professor at Imperial College London. He has been working in the area of organic polymeric electronic materials for the past 27 years.

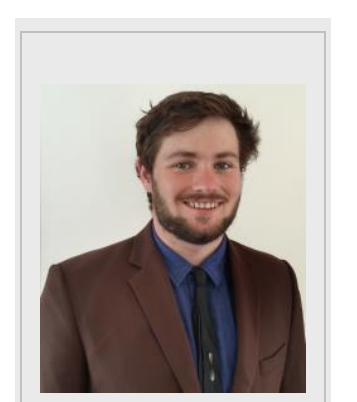

\section{Conjugated Polymers}

Conjugated polymers serve as a very popular class of materials in OPV devices. The properties of polymers can be easily tuned by varying the monomer building blocks. Indeed, the most often studied polymer structures consist of electron donating (D) and electron accepting $(A)$ monomers. Properties can be controlled by adjusting the electron affinity of the $D$ and $A$ units. Solution processability is another selling point for polymeric materials as the viscosity of polymer solutions enables deposition of uniform defect-free films. As mentioned in the introduction, readers can refer to a number of reviews on conjugated polymers in OPV devices for more comprehensive overview of the area. ${ }^{[4-10]}$ This section presents our group's insights into polymer design and synthesis and the effects on device performance..

Prior to 2008, most of the conjugated polymers synthesized in our group were used for organic light emitting diode (OLED) applications. While the polymers employed for OLED have some features in common with those used in OPV, the design of materials for OPV presented a different challenge. Apart from the need to tune the absorption profile of the polymers, it is essential to ensure that the frontier orbital energy levels are well matched between the electron donor and acceptor components of the BHJ. In addition, desirable semiconducting properties such as higher charge mobilities that are required in OPV materials are more difficult to realize. As a consequence, our group initially focused on optimization of polymerization methods to produce known conjugated polymers showing promising device performance.

\subsection{Continuous flow processing and scale-up}

A significant portion of our research program was devoted to device fabrication and testing. Studies included the fabrication of large area devices on flexible substrate using roll-to-roll printing techniques (Figure 2). ${ }^{[16-17]}$ Substantial quantities of materials were used for such experiments to optimize the printed devices. While some OPV materials, such as poly(3-hexylthiophene) P3HT, are commercially available at the scale required, we anticipated that it would be advantageous to have the ability to scale up the synthesis of materials, especially for ones developed in-house.
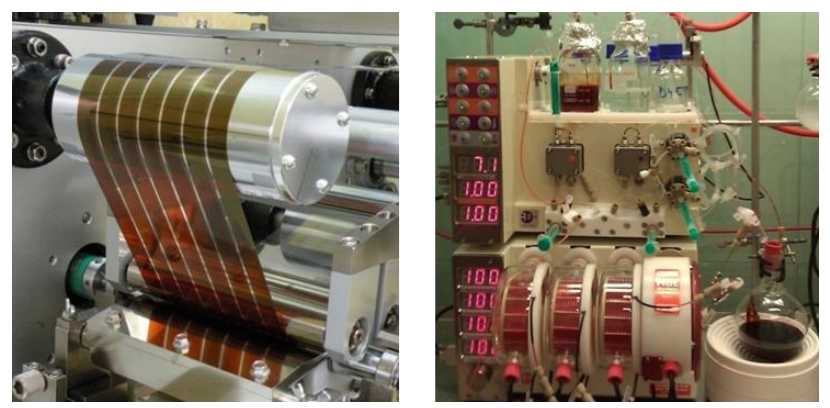

Figure 2. Picture of a roll-to-roll printer for OPV device fabrication on flexible substrate (left) and a continuous flow reactor ${ }^{[18]}$ (right). 
Compared with conventional synthesis methods, continuous flow processing is inherently scalable and it has additional benefits of more controlled reaction conditions (e.g. temperature, mixing, reagent stoichiometry, etc.) that can result in higher reproducibility and product yield. ${ }^{[19-20]}$ Our group was one of the first to examine flow methods for the synthesis of organic semiconducting materials. The flow synthesis of intermediate building blocks and molecular organic dyes ${ }^{[21]}$ as well as fullerene derivatives ${ }^{[22]}$ (vide infra) has been reported by our group. A picture of the flow reactor ${ }^{[18]}$ used in our laboratory is shown in Figure 2.

A variety of reactions for conjugated polymer synthesis in flow has been reported. ${ }^{[23-24]}$ Initially, the most commonly used reactions were examined. This included Suzuki polycondensation, Stille polycondensation and the synthesis of poly(phenylenevinylene) via the Gilch route. ${ }^{[23]}$ In general, it was found that polymerization under flow conditions shortened reaction time as a result of more efficient heat transfer and mixing. For the biphasic Suzuki polycondensation, improved mixing was a key factor in increasing the rate of the reaction. Stille polycondensation was also well suited to flow processing, as solutions can be heated under pressure (e.g. toluene at $170{ }^{\circ} \mathrm{C}$ ) much like the conditions used in microwave-assisted reactions. ${ }^{[25]}$

The successful translation of batch reaction conditions to flow processing relied on experimentation and accumulation of know-how. For instance, the flow synthesis experiment for polyfluorene PFO was relatively straightforward (Figure 3a). However, problems were encountered for the flow synthesis of PCDTBT (Figure 3b). It was found that the thienobenzothiadiazole monomer 3 a did not have sufficient solubility in the reaction media. This was not an issue in batch reactions as the reactants became more soluble as the reaction progressed. With the flow synthesis equipment available to us, reactants must be fully dissolved for the pumps to operate. This led to the use of an alternative monomer $\mathbf{3 b}$ for the successful synthesis of the analogous polymer PCDHTBT. Apart from the solubility of the reagents and reactants, the solubility of the product is also an important consideration. Poly(phenylene-vinylene)s have been known to form gels in solvents at certain molecular weights. To avoid blockage of the flow reactor, an additive was included in the reaction to mediate the polymerization. ${ }^{[23]}$

The synthesis of P3HT via Grignard metathesis polymerization (GRIM) was also demonstrated in flow processing (Figure 4). ${ }^{[24]}$ The reaction conditions were difficult to optimise because of the lack of stability of the thiophene Grignard monomers and the nickel catalyst species. After optimization, P3HT was successfully synthesized under flow conditions with molecular weight control (Figure 4b). ${ }^{[2]}$ Most recently, we reported direct arylation polymerization using flow methods. ${ }^{[26]}$ The use of inorganic carbonate bases in anhydrous organic solvents for these reaction meant that well-packed columns containing carbonate had to be used to accommodate the flow process. While we have demonstrated the possibility of flow processing in the synthesis of conjugated polymers, only multigram scale reactions were examined. However, we envisage that the flow methods developed will be critical in supporting efforts to upscale polymer solar cell fabrication in roll-to-roll processing. ${ }^{[27]}$

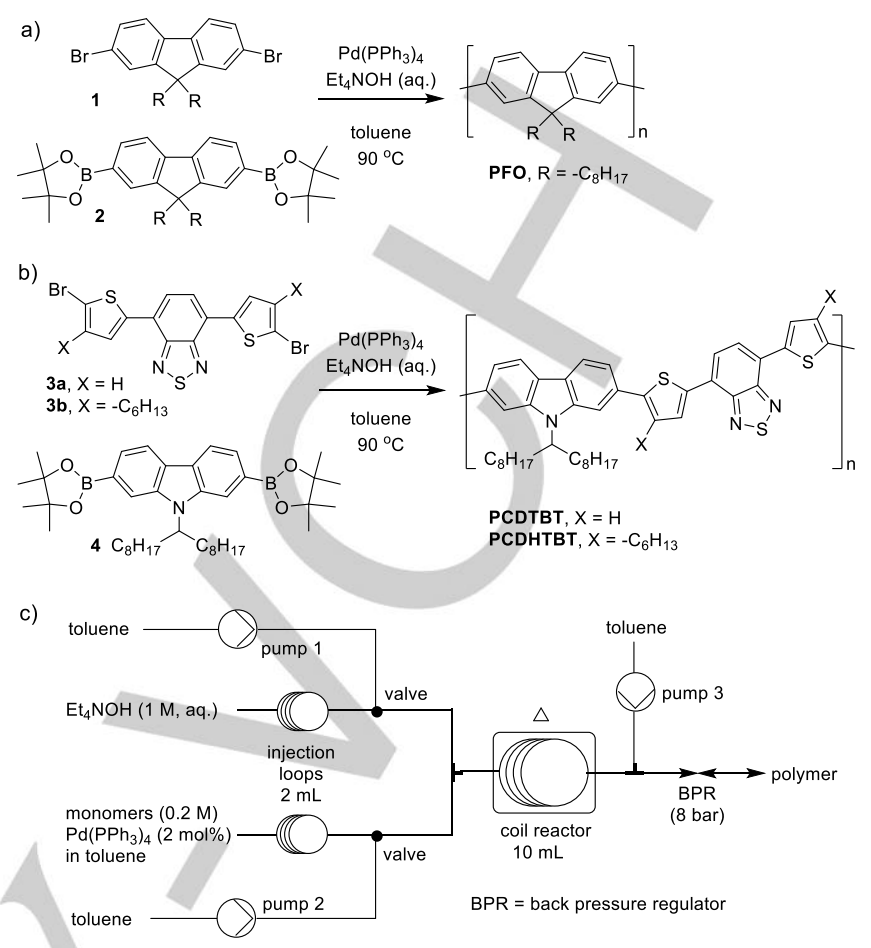

Figure 3. Polymer synthesis via Suzuki polycondensation for (a) PFO; (b) PCDHTBT and (c) schematic of the continuous flow reaction.

(a)

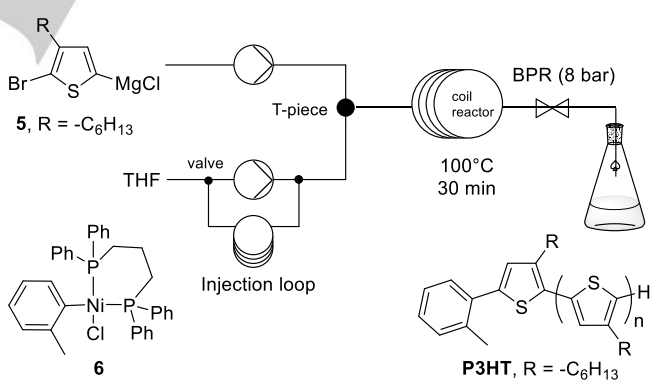

(b)

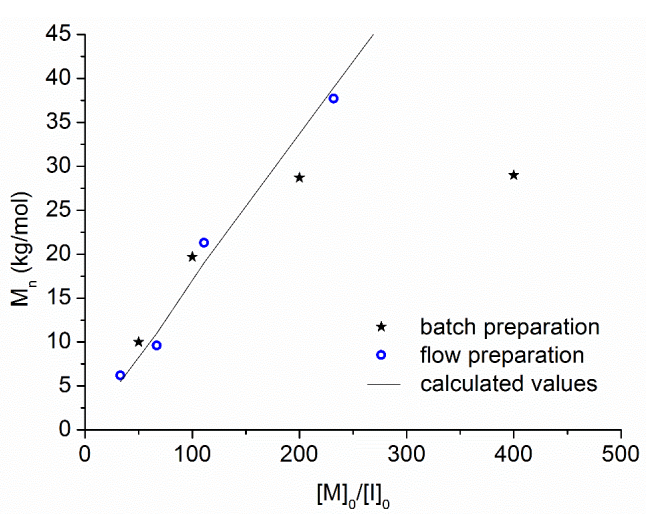

Figure 4. (a) Synthesis of P3HT in continuous flow from thiophene Grignard reagent $\mathbf{5}$ and nickel catalyst $\mathbf{6}$ and (b) the molecular weight, $M_{n}$, of the polyme scales linearly with monomer to catalyst ratio in both batch and flow reactions.

\subsection{Synthesis and molecular weight control}


At the same time as the development of flow processing methods, our group was also interested in optimization of batch reaction conditions to improve the quality of the polymeric materials. The general observation in the field has been that polymers with higher molecular weight range gave better performance in organic electronic devices. ${ }^{[28-31]}$ Our group has reported some examples of the effect of synthetic method on molecular weight and device performance. ${ }^{[32-33]}$

One example originated from the desire to translate conventional batch polymerization to flow processing. The polymer, PBDT-BT, was first synthesized by Stille polycondensation (Figure 5). ${ }^{[33]}$ A number average molecular weight $\left(M_{n}\right)$ of $40 \mathrm{~kg} / \mathrm{mol}$ was achieved using this coupling method and the material performance was promising in BHJ OPV devices ( $5 \%$ PCE). The scale-up of this material was considered using flow processing. It was soon apparent that the benzothiadiazole monomer building block 8 had poor solubility in the reaction medium. To circumvent this problem, Suzuki polycondensation was proposed with the use of the soluble boronic acid pinacol ester monomer 10 (Figure 5). This move was fortuitous as much higher molecular weight polymer $\left(M_{n} 112 \mathrm{~kg} / \mathrm{mol}\right)$ was isolated using Suzuki polycondensation. Even more remarkable was the fact that the high molecular weight material showed a substantially improved performance (8.5\%) and with further device optimization the PCE was increased to $9.4 \% .{ }^{[33]}$ This is almost double the performance of the polymer obtained by Stille polycondensation. ${ }^{[33]}$ However, it is interesting to note that Suzuki polycondensation also delivered an even higher molecular weight fraction $\left(M_{n} 136 \mathrm{~kg} / \mathrm{mol}\right)$ but resulted in a decrease in efficiency $(6.4 \%)$. This was attributed to the poor solubility of the large polymer that resulted in lower quality thin films. This highlights an interesting point concerning polymer semi-conductors. The unique chemical and physical properties of each polymer mean that a different optimal polymer size may be required for each material. However, this can also be seen as a limiting factor as rational chemical design cannot predict the desirable polymer size for OPV applications. It is interesting to note that the Suzuki polycondensation reaction was extremely fast for this system and the polymeric material became difficult to process in fewer than 30 minutes if the reaction was heated at $90^{\circ} \mathrm{C}$. At first glance, this appears to be the ideal reaction for a high performance material. However, the speed of the reaction meant that molecular weight outcome could vary significantly from batch to batch, resulting in reproducibility issues that are yet to be resolved.

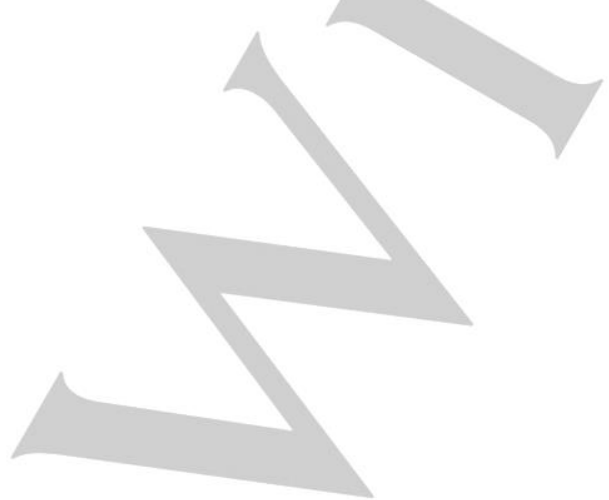

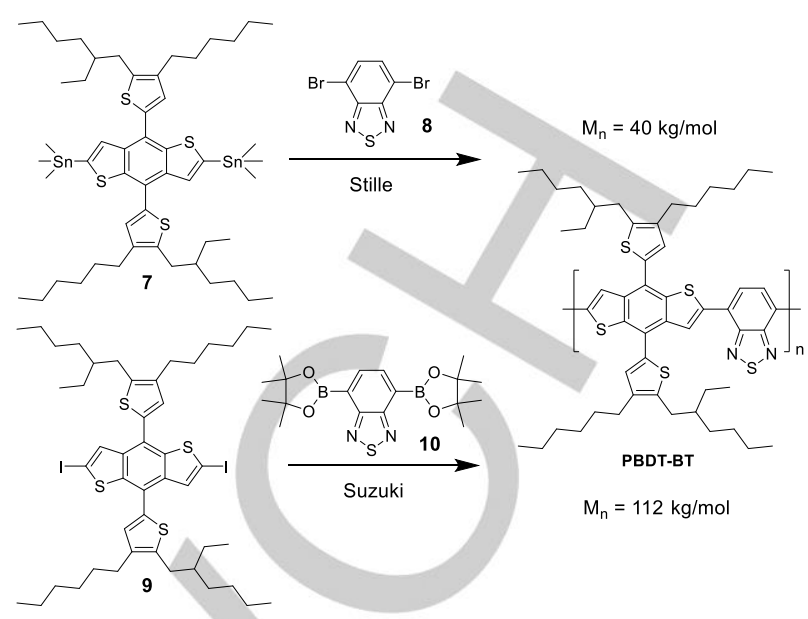

Figure 5. Synthesis of polymer PBDT-BT using Stille and Suzuki polycondensation methods giving samples of different molecular weight.

The dependence of device performance on molecular weight of the polymeric material was reported in another system synthesized using Stille coupling. ${ }^{[32]}$ Optimization of Stille polycondensation conditions led to a series of polymers with molecular weight $\left(M_{n}\right)$ varying from $10 \mathrm{~kg} / \mathrm{mol}$ to $139 \mathrm{~kg} / \mathrm{mol}$. The trend of higher molecular weight material giving higher device performance was observed in this case except for the polymer fraction at $139 \mathrm{~kg} / \mathrm{mol}$. At this very high $M_{n}$, the processability of the polymer was poor, resulting in problems during thin film deposition.

Lastly, our group has also observed decrease in device performance in polymeric materials made using different synthetic methods where variation of molecular weight played no role. It has been reported that the germole-based polymer, PDTGe-TPD, was used in devices with $>8 \%$ PCE. ${ }^{[34]}$ The synthesis of this polymer was re-examined by our group because it is generally acknowledged that the tin reagent used in the Stille coupling reaction has stability issues and was difficult to purify. The direct arylation polymerization approach was investigated and polymers of similar molecular weight to those from the Stille route were isolated. Most characterization data, including UV-vis absorption and redox properties, of the two polymer samples were very similar,but a large difference in device performance was recorded with the direct arylation polymer giving devices at $\sim 2 \%$ PCE. Interestingly, the ${ }^{1} \mathrm{H}$ NMR spectrum of the polymers revealed some differences. The variable temperature NMR experiment suggested that the polymer strongly aggregated in solution at room temperature (Figure 6). By comparing the integration of the resonances assigned to the proton on the polymer backbone in the aggregated and 'free' state, it was possible to deduce that the polymer produced by direct arylation did not aggregate as strongly as the one prepared by Stille coupling. While no direct evidence has as yet been obtained, we speculate that there are more defect sites on the direct arylation polymer, therefore affecting aggregation. It has been reported previously that carbon-carbon bond formation by direct arylation can produce regioisomer and homo-coupling defects on a polymer backbone. ${ }^{[35-37]}$ 
(a)

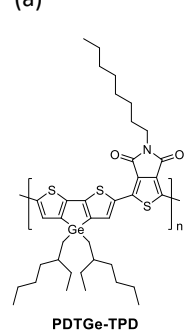

(b)
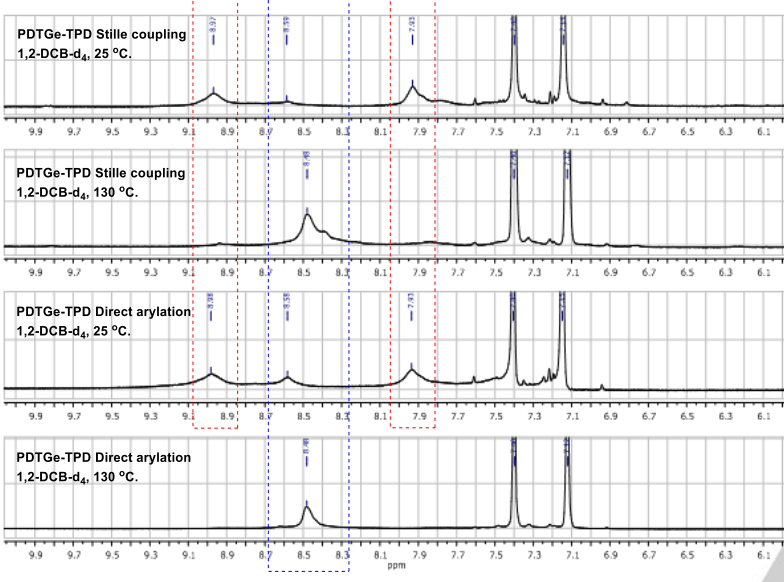

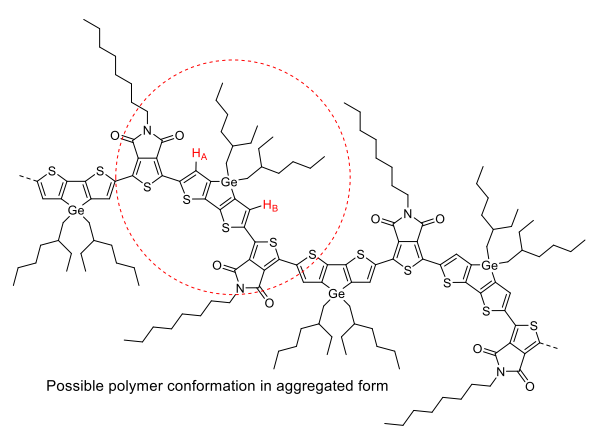

\section{.} LUMO energies relative to the homopolymer P9 while the HOMO energies of the polymers remain constant. Interestingly, polymers $\mathbf{P 9}$ and $\mathbf{P 1 0}$ have quantum yields exceed $80 \%$. The combination of the $\mathrm{BTz}$ unit with the highly electron deficient benzobisthiadiazole (BBT) monomer gave a blue polymer with absorption onset at $700 \mathrm{~nm}$. The BTz-BBT polymer P12 was used as the electron acceptor material in OPV devices with P3HT as donor material, which showed moderate performance of $0.4 \%$ PCE due to unfavorable phase separation between P3HT and P12.

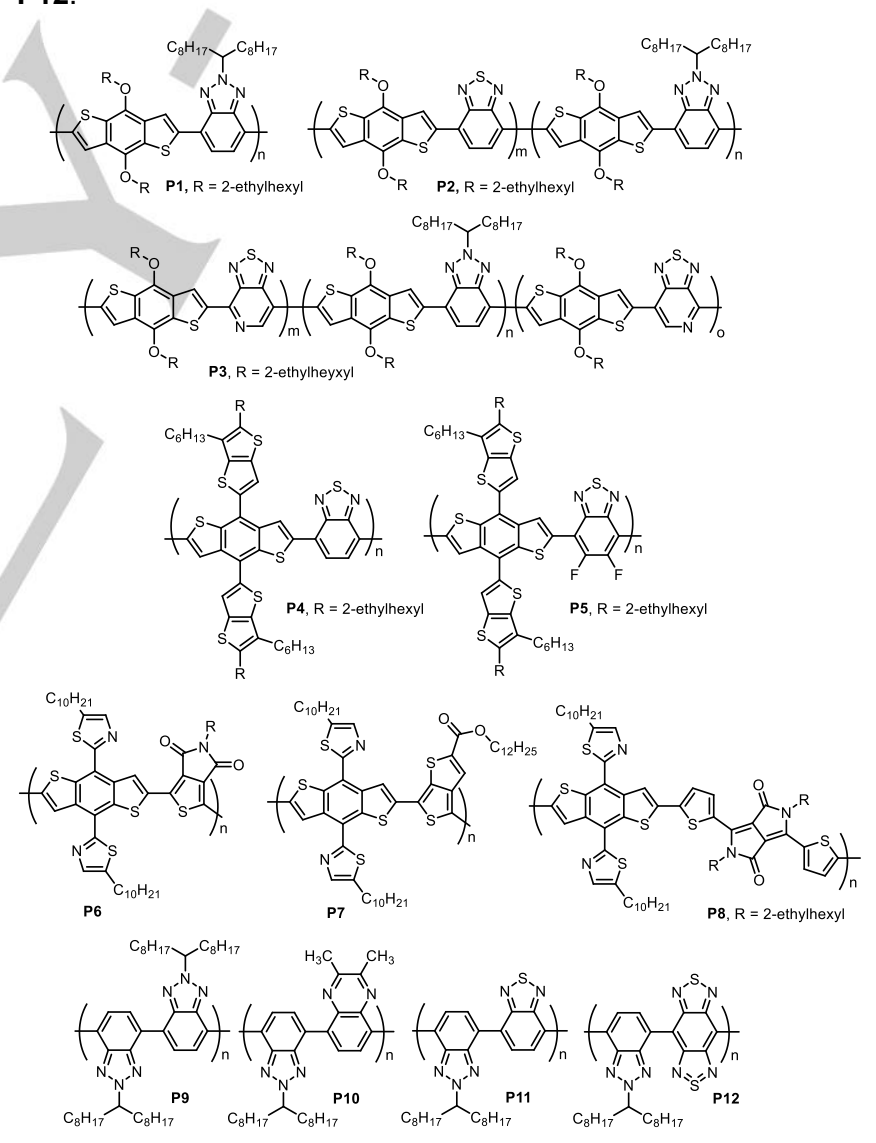

Figure 7. Structures of polymers used in OPV devices.

\section{Molecular Materials} direction ortogonal to the polymer backone, thienothiophene was incorporated (Figure 7, P4 and P5). ${ }^{[39]}$ Thiazole was also

substituted on the BDT unit (Figure 7, P6-P8). ${ }^{[40]}$ Thiazole is nown to be electron deficient compared to thiophene. The perties of the resulting polymers were a little unexpected as orbital energy levels of the polymers. The photovoltaic performances of all BDT derivatives as donor polymers is highly $\mathrm{PC}_{71} \mathrm{BM}$. Conjugated polymers containing electron deficient building blocks only has also been systematically investigated by using a weak electron deficient core, $2 \mathrm{H}$-benzotriazole (Figure 7 , P9-P12).$^{[41]}$ Increasing acceptor strength resulted to shifts in the

Figure 6. (a) Chemical structure of polymer PDTGe-TPD and possible polyme backbone configuration in aggregated form and (c) variable temperature ${ }^{1} \mathrm{H}$ NMR spectrum of the polymer synthesized by Stille coupling and direct arylation at 25 and $130^{\circ} \mathrm{C}$ in 1,2-dichlorobenzene- $d_{4}$.

\subsection{Polymer structure}

Like many other researchers, our group has also examined the effect of modification of the polymer structure on properties and device performance. Benzodithiophene (BDT) is a very popular electron donating building block in polymers for OPV devices. ${ }^{[5]}$ In one study, a series of polymers was synthesized using mixtures of electron acceptor monomers with the BDT building block (Figure 7, P1-P3). ${ }^{[38]}$ By increasing the electron acceptor strength from benzotriazole (BTz) to benzothiadiazole (BT) to pyridylthiadiazole (РyT), the absorption edge of the polymers was extended from $600 \mathrm{~nm}$ to $750 \mathrm{~nm}$. The use of BTz with BT or PyT broadened the absorption of the polymer with coverage over a large portion of the visible range. The BTz unit with branched alkyl substituents also improved the solubility and solution processability of the polymers. Interestingly, the BDT-BT polymer P1 showed remarkable molecular order in bulk samples prepared by fiber extrusion. ${ }^{[38]}$ The analysis of the polymer structure in this sample is currently under investigation using a combination of wide angle X-ray scattering and solid state NMR experiments.

The 3,6-positions of the benzene ring in the BDT core are easily accessible for facile modulation of the electronic properties
Our group has been interested in molecular materials since we started research into the OPV area. Compared with polymers, the 
structure of molecular materials is defined, and higher purity samples can be achieved. The consequence of this is that devices based on molecular materials can have greater stability and reproducibility. The deposition of molecular materials can be achieved by solution processing as well as by thermal evaporation under vacuum. Again, readers are directed to reviews in the area for comprehensive overview as we will focus on our personal experience and insight in this account. ${ }^{[11-13]}$

\subsection{Hexa-peri-hexabenzocoronene (HBC)}

The defined structure of molecular materials is particularly attractive for studies in intermolecular association and its effect on bulk material properties and device characteristics. One molecular system that drew our attention is hexa-perihexabenzocoronene (HBC) ${ }^{\left[{ }^{[2]}\right]} \mathrm{HBC}$ is a class of polycyclic aromatic hydrocarbons that has a very strong propensity to associate through $\pi-\pi$ interactions. A number of research groups have taken advantage of this property and used the material to form interesting self-assembled structures for a variety of applications. ${ }^{[42]}$ Inspired by some early work, our focus has been to use HBCs in organic electronics, in particular OPV devices. The HBC core without substitution is insoluble and can only be processed by thermal evaporation. As such a large number of studies have reported on the functionalization of the periphery of the HBC molecule. Our contribution to the area is the use of fluorene groups to impart solubility and, at the same time, provide a handle for further functionalization (Figure 8). ${ }^{[42]}$

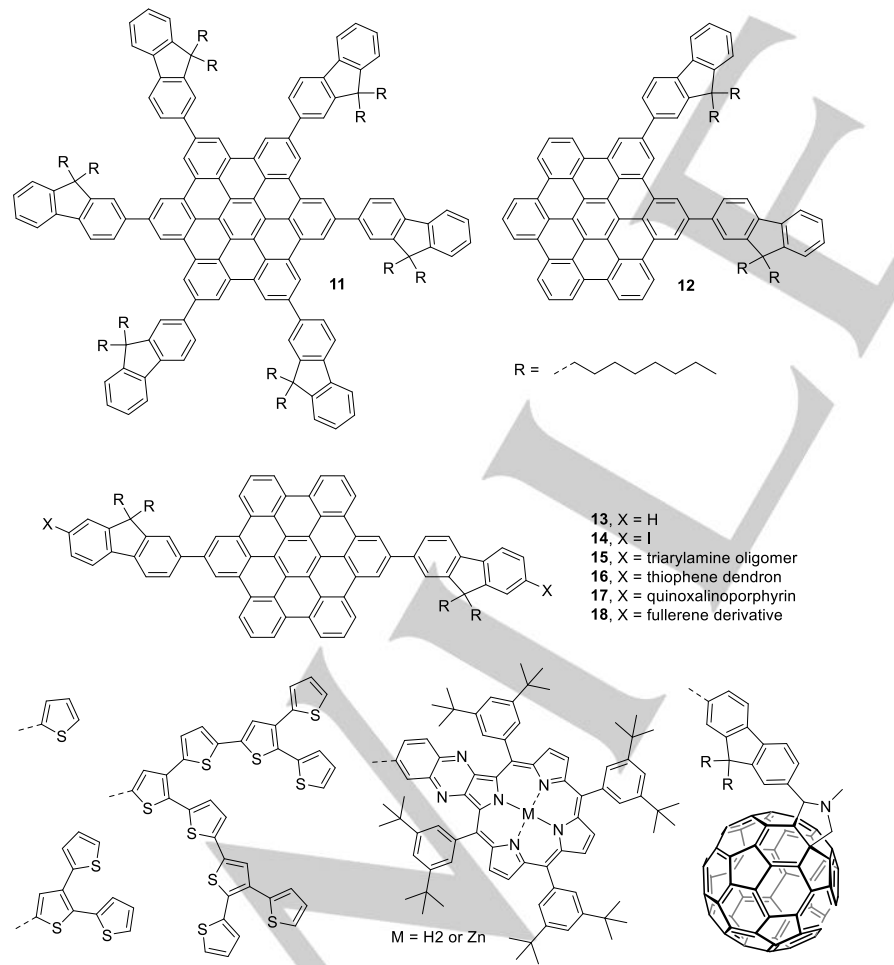

Figure 8. Structures of FHBC derivatives.

Fluorenyl HBC (FHBC) derivatives showed self-association behavior typical of HBC compounds. Association through $\pi-\pi$ stacking drives the assembly of columnar aggregates in solution and in solid state. Evidence for this was observed in ${ }^{1} \mathrm{H}$ NMR spectroscopy (Figure 9) and wide-angle X-ray scattering experiments respectively (Figure 10). The feature that separates FHBC derivatives from other HBC compounds is charge transport pathway made possible by the fluorene groups connecting the cores of the HBC columnar assembly. The result is improved charge transport and enhanced semiconducting properties for the FHBC materials. ${ }^{[43]}$ This led to OPV devices with PCE of $1.5 \%$ despite the less than ideal spectral coverage of the FHBC material 13. Attempts to improve the performance of FHBC materials were made by adding a variety of chromophores to enhance light absorption. ${ }^{[44-48]}$ Up to $3 \%$ PCE was observed but there was a trade-off between the spectral coverage of the material and its semiconducting properties. Essentially, greater absorption was achieved with substituents, such as thiophene dendrons ${ }^{[47]} 16$ and porphyrins ${ }^{[48]} 17$, but the steric bulk of these units disrupted the molecular order of the bulk material leading to poorer charge transport. Apart from extending the absorption profile of these materials, the HBC motif has also been used to build ambipolar materials with fullerene derivatives. ${ }^{[49]}$ Single component active layer OPV devices were fabricated and maximum efficiency of $0.22 \%$. While the close proximity of the electron donor and acceptor units was advantageous for charge separation, the possibility of charge recombination was also higher in these structures. For a more detail discussion on the design, synthesis and OPV application of FHBC derivatives, readers can refer to a review article. ${ }^{[42]}$

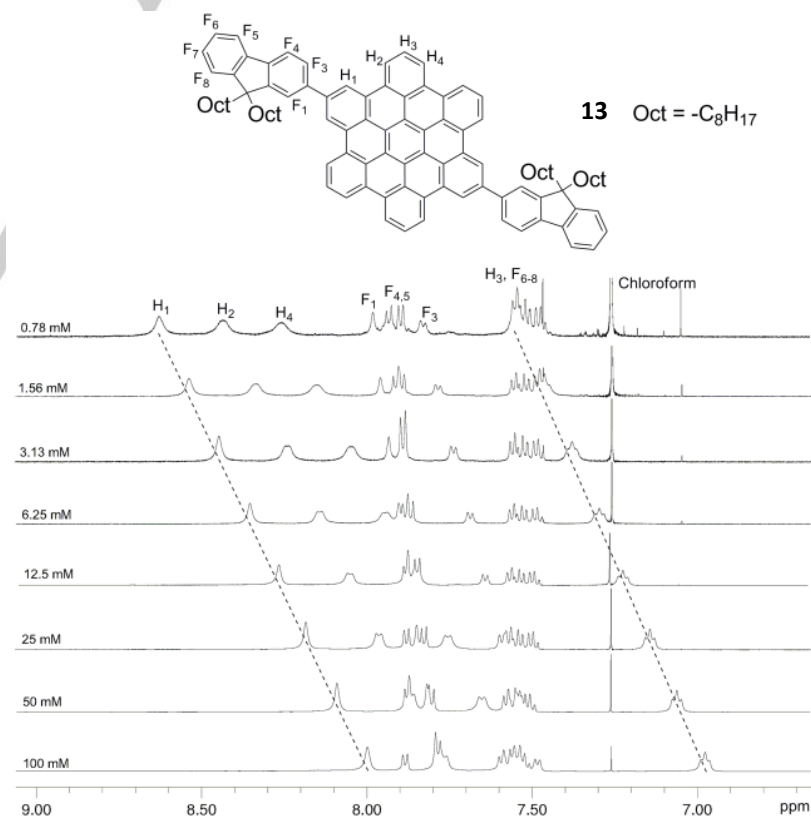

Figure 9. The ${ }^{1} \mathrm{H}$ NMR spectrum of $\mathrm{FHBC}$ compound 13 in $\mathrm{CDCl}_{3}$ at a range of concentrations. The up field shift for the HBC core protons with increasing concentration is a result of the ring current effect due to the $\pi-\pi$ stacking of the molecules. 
(a)

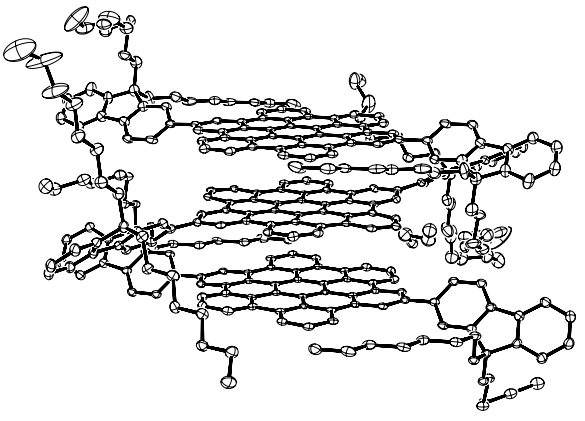

(b)
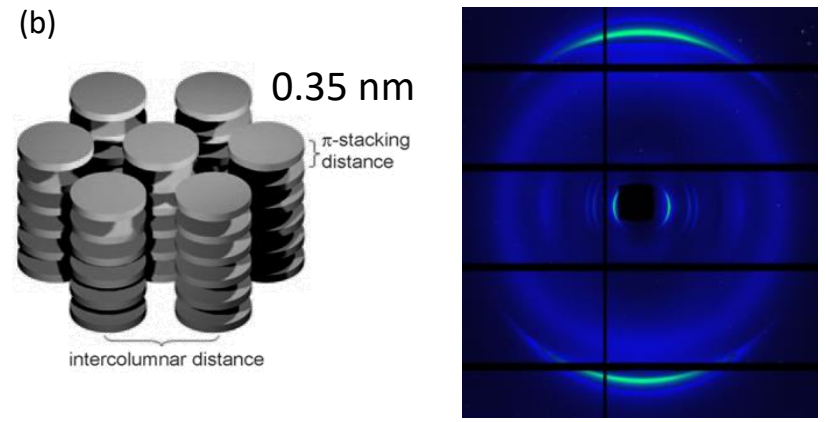

Figure 10. (a) Thermal ellipsoid illustration of the crystal structure of compound 13 and (b) two-dimensional wide angle X-ray scattering data indicating columnar structures in extruded fibre samples.

FHBC compounds have been used as an interface modifier. ${ }^{[50]}$ Molecules containing amphiphilic groups were designed to be deposited on surfaces or to accumulate at hydrophilic/hydrophobic interfaces. As a result of the strong supramolecular interactions in HBC assemblies, it was possible to use these interface materials to promote bulk structure changes in organic films. While there is some way to go before control of film microstructure can be realized, we showed that the use of interface modifiers is a viable strategy going forward. We believe that using molecular structural changes to tune bulk material structure and properties is a better strategy than the use processing techniques, such as solution sheering, thermal annealing, solvent annealing, etc.

\subsection{Semiconducting dyes}

Apart from $\pi-\pi$ stacking, other supramolecular association forces can also be important in OPV materials. The role of hydrogen bonding in a series of oligothiophene and fullerene derivatives was investigated. ${ }^{[51]}$ Hydrogen bonding had a significant effect on the bulk film structure of the materials. In our case, the strong association between molecules led to greater aggregation. This meant that there was larger electron donor and electron acceptor domains in BHJ films. This study highlighted the need to consider carefully not just the interactions of one material, but also the interactions in material blends.

In organic materials, weak dispersion forces such as van der Waal interactions can also make significant changes to bulk material properties. An oligothiophene material, BTR, was reported recently showing high performance (>9\% PCE) in OPV devices. ${ }^{[52]}$ The core structure of BTR has been used previously by other groups and excellent performance of 8 to $9 \%$ was recorded. ${ }^{[2,53-54]}$ By positioning the alkyl side chains in a 'regioregular' manner, the thermal behavior and crystallinity of the BTR compound are somewhat different from analogs in the literature. A liquid crystalline transition was observed at $186^{\circ} \mathrm{C}$ for the BTR material (Figure 11). Further studies are in progress to investigate the effects of side chain modification on this oligothiophene system.

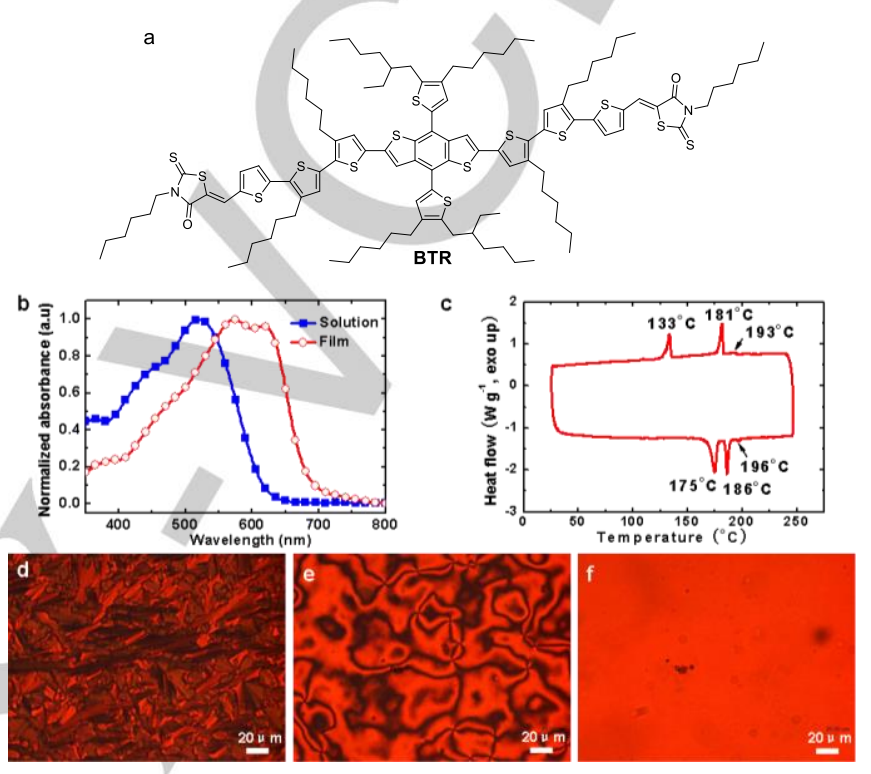

Figure 11. Molecular structure, optical absorption spectra, DSC thermogram and POM images. (a) Chemical structure of BTR. (b) Normalized UV-vis absorption spectra of BTR in chloroform $(5 \mathrm{mg} / \mathrm{mL})$ and in spin-cast film. (c) differential scanning calorimetry thermogram of BTR in nitrogen at a ramp rate of $10^{\circ} \mathrm{C} \mathrm{min}^{-1}$. The lower trace is from heating cycle and upper trace from cooling cycle. (d) BTR thin film sandwiched in between two glass slides observed under a polarized optical microscope at a stage temperature of $185^{\circ} \mathrm{C}$, (e) $195^{\circ} \mathrm{C}$ and (f) $197^{\circ} \mathrm{C}$

\subsection{Fullerene acceptors}

Fullerenes $\left(\mathrm{C}_{60}\right.$ and $\left.\mathrm{C}_{70}\right)$ and their derivatives have been successfully used in OPV devices since the 1990s. Phenyl- $\mathrm{C}_{61}$ butyric acid methyl ester $\left(\mathrm{PC}_{61} \mathrm{BM}\right)$ and its $\mathrm{C}_{70}$ analogue $\left(\mathrm{PC}_{71} \mathrm{BM}\right)$ are still the most popular electron acceptor component in $\mathrm{BHJ}$ OPV devices. ${ }^{[55-56]}$ There has been many studies to tune the photophysical properties of fullerene derivatives but few have surpassed the performance of $\mathrm{PC}_{61} \mathrm{BM}$ and $\mathrm{PC}_{71} \mathrm{BM}$.

One successful example is the use of fullerene bis-adducts in devices. As the open circuit voltage ( $\left.V_{o c}\right)$ of a device is directly related to the HOMO energy level of the electron donor material and the LUMO energy level of the electron acceptor material, adjusting the LUMO energy level of the fullerene derivative can provide a boost to device performance. With two substituents on the fullerene molecule, the material is less electron deficient than the mono substituted compounds leading to an increase in LUMO energy. The result is a larger $V_{o c}$, which can increase the overall efficiency of the device. For example, the indene-fullerene 
bisadducts (ICBA) gave devices with very good PCE of $>7 \%$ for both the $\mathrm{C}_{60}$ and $\mathrm{C}_{70}$ analogues. ${ }^{[57-58]}$

The high performance for ICBA can be achieved despite the fact that the materials are mixtures of regioisomers. It was considered interesting to investigate the material properties and device performance of ICBA samples that consist of a single isomer. Our group has reported the chromatographic isolation of a single isomer of $\mathrm{IC}_{70} \mathrm{BA}$ using a combination of silica and nitrocarbazole-functionalized solid phases. ${ }^{[59]}$ An interesting aside is the fact that this work developed serendipitously from scale-up experiments involving flow processing. ${ }^{[22]}$ In the multi-gram scale synthesis of $\mathrm{IC}_{70} \mathrm{BA}$, we found that the isomer mixture can be separated into two fractions using flash chromatography on silica. This set us on the path of isolating the single isomer. Single crystal X-ray data provided the relative configuration of the isomer (Figure 12). When compared with isomer mixtures, the single isomer material showed higher electron transport mobility and improved performance in OPV devices. We are currently in the process of completing the analysis of other regioisomers from the $\mathrm{IC}_{70} \mathrm{BA}$ mixture.

Going forward, it is uneconomical, both in chemicals required and time, to use a material that requires chromatographic isolation. Tether directed remote functionalization has been used previously to control the regioselectivity of fullerene multi-adduct formation. ${ }^{[60-62]}$ We have recently used this synthetic strategy to obtain a $\mathrm{C}_{60}$ bisadduct single isomer from $\mathrm{PC}_{61} \mathrm{BM}$ in one pot over two steps. ${ }^{[63]}$ The performance of this material compared favorably with both $\mathrm{PC}_{61} \mathrm{BM}$ and $\mathrm{a}_{60}$ bisadduct isomer mixture.

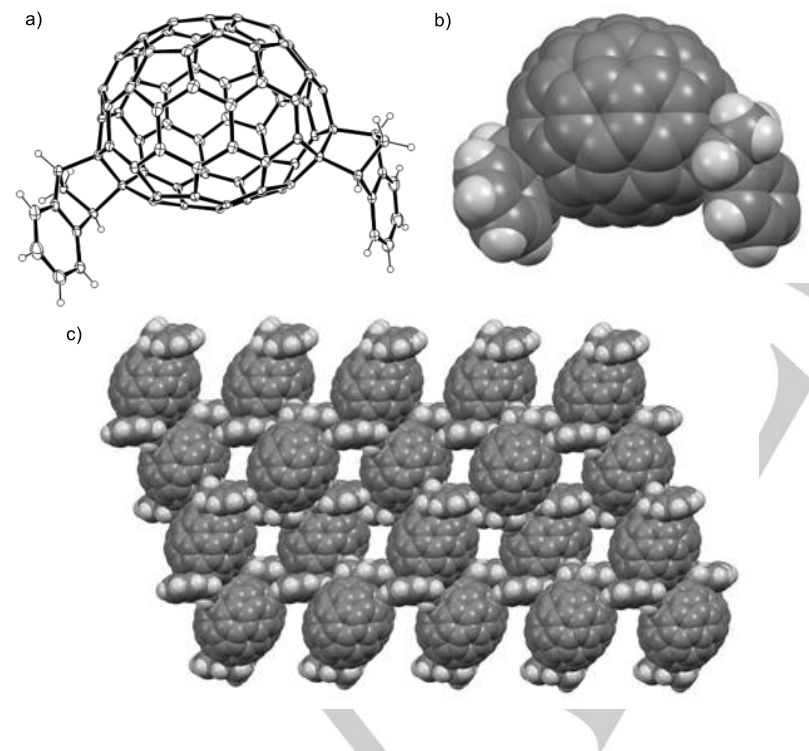

Figure 12. Structure obtained by $X$-ray analysis of a single isomer of $I_{70} B A$. (a) Thermal ellipsoid illustration, (b) space filling model, and (c) packing diagram where disordered $\mathrm{CHCl}_{3}$ solvent molecules have been omitted for clarity.

\section{Summary and Outlook}

In this account, we have shared our experience and insight into the development of materials for OPV devices. In all the examples provided, it is clear that an intimate connection between material design, synthesis, characterization and device testing is of paramount importance for a successful outcome. It is also apparent that there is still a substantial gap in understanding the relationship between molecular structure and properties, bulk material properties and device performance. Further experiments and additional examples are required to shed more light on this subject. In the not-too-distant future, analysis of material libraries will result in tangible outcomes and advancement for the field of OPV. In addition to materials design and applications, we learnt a valuable lesson in the continuous flow synthesis studies. The primary aim of the flow experiments was to demonstrate material scale-up capability which was shown to be possible for a wide range of reactions commonly used in organic electronic materials synthesis. In doing so, the synthesis and properties of materials were carefully re-examined ultimately resulting in device performance enhancements. There is still significant scope to further develop flow processing in materials chemistry.

\section{Acknowledgements}

The bulk of the work described in this account was funded by the Australian Renewable Energy Agency (ARENA) and the Victorian State Government. WWHW currently receives funding from ARENA through the Australian Centre for Advanced Photovoltaics and from the Australian Research Council Future Fellowship Scheme (FT130100500). JLB, PBG and QH are supported by Australian Postgraduate Awards.

Keywords: organic photovoltaics $•$ conjugated polymers polycyclic aromatic hydrocarbons $\cdot$ fullerene derivatives • continuous flow processing

[1] Y. Liu, J. Zhao, Z. Li, C. Mu, W. Ma, H. Hu, K. Jiang, H. Lin, H. Ade, H. Yan, Nat. Commun. 2014, 5, 5293.

[2] Y. Liu, C.-C. Chen, Z. Hong, J. Gao, Y. Yang, H. Zhou, L. Dou, G. Li, Y. Yang, Sci. Rep. 2013, 3, 3356.

[3] J. You, L. Dou, K. Yoshimura, T. Kato, K. Ohya, T. Moriarty, K. Emery, C.-C. Chen, J. Gao, G. Li, Y. Yang, Nat. Commun. 2013, 4, 1446.

[4] H. Zhou, L. Yang, W. You, Macromolecules 2012, 45, 607632.

[5] Y. Li, Acc. Chem. Res. 2012, 45, 723-733.

[6] D. Gendron, M. Leclerc, Energy Environ. Sci. 2011, 4 1225-1237.

[7] A. Facchetti, Chem. Mater. 2011, 23, 733-758.

[8] X. Zhan, D. Zhu, Polym. Chem. 2010, 1, 409-419.

[9] C. J. Brabec, S. Gowrisanker, J. J. M. Halls, D. Laird, S. J. Jia, S. P. Williams, Adv. Mater. 2010, 22, 3839-3856.

[10] Z.-G. Zhang, Y. Li, Sci. China Chem. 2015, 58, 192-209.

[11] A. Mishra, P. Bäuerle, Angew. Chem. Int. Ed. 2012, 51, 2020-2067.

[12] Y. Lin, Y. Li, X. Zhan, Chem. Soc. Rev. 2012, 41, 42454272.

[13] B. Walker, C. Kim, T.-Q. Nguyen, Chem. Mater. 2010, 23 , 470-482.

[14] Z. B. Henson, K. Müllen, G. C. Bazan, Nat. Chem. 2012, 4, 699-704. 
[15] K. Sun, Z. Xiao, E. Hanssen, M. F. G. Klein, H. H. Dam, M. Pfaff, D. Gerthsen, W. W. H. Wong, D. J. Jones, J. Mater Chem. A 2014, 2, 9048-9054.

[16] J. Yang, D. Vak, N. Clark, J. Subbiah, W. W. H. Wong, D. J. Jones, S. E. Watkins, G. Wilson, Sol. Energy Mater. Sol. Cells 2013, 109, 47-55.

[17] D. Vak, J. van Embden, W. W. H. Wong, S. Watkins, Appl. Phys. Lett. 2015, 106, 033302.

[18] http://www.vapourtec.co.uk/

[19] R. L. Hartman, J. P. McMullen, K. F. Jensen, Angew. Chem. Int. Ed. 2011, 50, 7502-7519.

[20] G. Jas, A. Kirschning, Chem. Eur. J. 2003, 9, 5708-5723.

[21] H. Seyler, S. Haid, T.-H. Kwon, D. J. Jones, P. Bäuerle, A. B. Holmes, W. W. H. Wong, Aust. J. Chem. 2013, 66, 151156.

[22] H. Seyler, W. W. H. Wong, D. J. Jones, A. B. Holmes, J. Org. Chem. 2011, 76, 3551-3556.

[23] H. Seyler, D. J. Jones, A. B. Holmes, W. W. H. Wong, Chem. Commun. 2012, 48, 1598-1600.

[24] H. Seyler, J. Subbiah, D. J. Jones, A. B. Holmes, W. W. H. Wong, Beilstein J. Org. Chem. 2013, 9, 1492-1500.

[25] R. C. Coffin, J. Peet, J. Rogers, G. C. Bazan, Nat. Chem. 2009, 1, 657-661.

[26] F. Grenier, B. R. Aïch, Y.-Y. Lai, M. Guérette, A. B. Holmes, Y. Tao, W. W. H. Wong, M. Leclerc, Chem. Mater. 2015, 27, 2137-2143.

[27] F. C. Krebs, T. Tromholt, M. Jorgensen, Nanoscale 2010, 2, 873-886.

[28] T.-Y. Chu, J. Lu, S. Beaupré, Y. Zhang, J.-R. Pouliot, J. Zhou, A. Najari, M. Leclerc, Y. Tao, Adv. Funct. Mater. 2012, 22, 2345-2351.

[29] J. J. Intemann, K. Yao, H.-L. Yip, Y.-X. Xu, Y.-X. Li, P.-W. Liang, F.-Z. Ding, X. Li, A. K. Y. Jen, Chem. Mater. 2013, 25, 3188-3195

[30] W. Li, L. Yang, J. R. Tumbleston, L. Yan, H. Ade, W. You, Adv. Mater. 2014, 26, 4456-4462.

[31] C. Liu, K. Wang, X. Hu, Y. Yang, C.-H. Hsu, W. Zhang, S. Xiao, X. Gong, Y. Cao, ACS Appl. Mater. Interfaces 2013 5, $12163-12167$.

[32] Z. Xiao, K. Sun, J. Subbiah, T. Qin, S. Lu, B. Purushothaman, D. J. Jones, A. B. Holmes, W. W. H. Wong, Polym. Chem. 2015, 6, 2312-2318.

[33] J. Subbiah, B. Purushothaman, M. Chen, T. Qin, M. Gao, D. Vak, F. H. Scholes, X. Chen, S. E. Watkins, G. J. Wilson, A. B. Holmes, W. W. H. Wong, D. J. Jones, Adv. Mater. 2015, 27, 702-705.

[34] C. E. Small, S. Chen, J. Subbiah, C. M. Amb, S.-W. Tsang, T.-H. Lai, J. R. Reynolds, F. So, Nat. Photon. 2012, 6, 115120.

[35] F. Lombeck, H. Komber, S. I. Gorelsky, M. Sommer, ACS Macro Lett. 2014, 3, 819-823.

[36] A. E. Rudenko, B. C. Thompson, J. Polym. Sci., Part A: Polym. Chem. 2015, 53, 135-147.

[37] S. Kowalski, S. Allard, U. Scherf, Macromol. Rapid Commun. 2015, DOI: 10.1002/marc.201400557.

[38] W. W. H. Wong, J. Subbiah, S. R. Puniredd, W. Pisula, D. J. Jones, A. B. Holmes, Polym. Chem. 2014, 5, 12581263.

[39] Z. Xiao, J. Subbiah, K. Sun, D. J. Jones, A. B. Holmes, W. W. H. Wong, Polym. Chem. 2014, 5, 6710-6717.

[40] Z. Xiao, J. Subbiah, K. Sun, S. Ji, D. J. Jones, A. B. Holmes, W. W. H. Wong, J. Mater. Chem. C 2014, 2, 1306-1313.
[41] J. L. Banal, J. Subbiah, H. Graham, J.-K. Lee, K. P. Ghiggino, W. W. H. Wong, Polym. Chem. 2013, 4, 10771083.

[42] H. Seyler, B. Purushothaman, D. J. Jones, A. B. Holmes, W. W. H. Wong, Pure Appl. Chem. 2012, 84, 1047-1067.

[43] W. W. H. Wong, T. B. Singh, D. Vak, W. Pisula, C. Yan, X. L. Feng, E. L. Williams, K. L. Chan, Q. Mao, D. J. Jones, C.-Q. Ma, K. Müllen, P. Bäuerle, A. B. Holmes, Adv. Funct. Mater. 2010, 20, 927-938.

[44] W. W. H. Wong, J. Subbiah, S. R. Puniredd, B Purushothaman, W. Pisula, N. Kirby, K. Müllen, D. J. Jones, A. B. Holmes, J. Mater. Chem. 2012, 22, 21131 21137.

[45] S. Ren, C. Yan, D. Vak, D. J. Jones, A. B. Holmes, W. W. H. Wong, Adv. Funct. Mater. 2012, 22, 2015-2026.

[46] W. W. H. Wong, C.-Q. Ma, W. Pisula, A. Mavrinskiy, X. Feng, H. Seyler, D. J. Jones, K. Müllen, P. Bäuerle, A. B. Holmes, Chem. Eur. J. 2011, 17, 5549-5560.

[47] W. W. H. Wong, C.-Q. Ma, W. Pisula, C. Yan, X. L. Feng D. J. Jones, K. Müllen, R. A. Janssen, P. Bäuerle, A. B. Holmes, Chem. Mater. 2010, 22, 457-466.

[48] W. W. H. Wong, T. Khoury, D. Vak, C. Yan, D. J. Jones, M. J. Crossley, A. B. Holmes, J. Mater. Chem. 2010, 20, 7005-7014.

[49] W. W. H. Wong, D. Vak, T. B. Singh, S. J. Ren, C. Yan, D. J. Jones, Liaw, II, R. N. Lamb, A. B. Holmes, Org. Lett. 2010, 12, 5000-5003.

[50] H. H. Dam, K. Sun, E. Hanssen, J. M. White, T. Marszalek, W. Pisula, J. Czolk, J. Ludwig, A. Colsmann, M. Pfaff, D. Gerthsen, W. W. H. Wong, D. J. Jones, ACS Appl. Mater Interfaces 2014, 6, 8824-8835.

[51] Z. Xiao, K. Sun, J. Subbiah, S. Ji, D. J. Jones, W. W. H. Wong, Sci. Rep. 2014, 4, 5701.

[52] K. Sun, Z. Xiao, S. Lu, W. Zajaczkowski, W. Pisula, E. Hanssen, J. M. White, R. M. Williamson, J. Subbiah, J. Ouyang, A. B. Holmes, W. W. H. Wong, D. J. Jones, Nat. Commun. 2015, 6, 6013.

[53] B. Kan, Q. Zhang, M. Li, X. Wan, W. Ni, G. Long, Y. Wang X. Yang, H. Feng, Y. Chen, J. Am. Chem. Soc. 2014, 136, 15529-15532.

[54] J. Zhou, Y. Zuo, X. Wan, G. Long, Q. Zhang, W. Ni, Y. Liu, Z. Li, G. He, C. Li, B. Kan, M. Li, Y. Chen, J. Am. Chem. Soc. 2013, 135, 8484-8487.

[55] Y. Li, Chem. Asian J. 2013, 8, 2316-2328.

[56] Y. He, Y. Li, Phys. Chem. Chem. Phys. 2011, 13, 19701983.

[57] S.-H. Liao, Y.-L. Li, T.-H. Jen, Y.-S. Cheng, S.-A. Chen, J. Am. Chem. Soc. 2012, 134, 14271-14274.

[58] X. Guo, C. Cui, M. Zhang, L. Huo, Y. Huang, J. Hou, Y. Li, Energy Environ. Sci. 2012, 5, 7943-7949.

[59] W. W. H. Wong, J. Subbiah, J. M. White, H. Seyler, B. Zhang, D. J. Jones, A. B. Holmes, Chem. Mater. 2014, 26, 1686-1689.

[60] W. W. H. Wong, F. Diederich, Chem. Eur. J. 2006, 12, 3463-3471.

[61] S. Sergeyev, F. Diederich, Angew. Chem. Int. Ed. 2004, 43, 1738-1740.

[62] M. J. van Eis, P. Seiler, L. A. Muslinkina, M. Badertscher E. Pretsch, F. Diederich, R. J. Alvarado, L. Echegoyen, I. Pérez Núñez, Helv. Chim. Acta 2002, 85, 2009-2055.

[63] B. Zhang, J. Subbiah, Y.-Y. Lai, J. M. White, D. J. Jones, W. W. H. Wong, Chem. Commun. 2015, DOI: 10.1039/c1035cc02701b. 
Entry for the Table of Contents (Please choose one layout)

Layout 1:

\section{PERSONAL ACCOUNT}

Experience and insight into the research on organic photovoltaic materials are presented in this account.

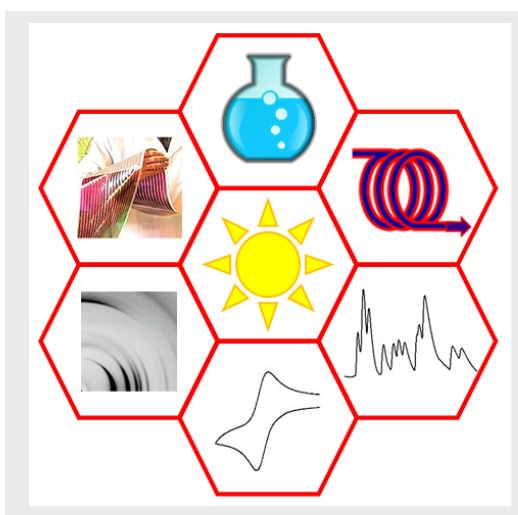

Wallace W. H. Wong, * James L. Banal, Paul B. Geraghty, Quentin Hong, Bolong Zhang, Andrew B. Holmes, David J. Jones

Page No. - Page No.

Organic Photovoltaic Materials Design, Synthesis and Scale-up 